\title{
Cytokine profile of diabetes-prone bb/ok rats and their congenic derivatives without diabetes or with reduced diabetes frequency
}

\begin{abstract}
Type 1 diabetes (T1D) is a chronic autoimmune disease in which an imbalance between pro- and anti-inflammatory and/or regulatory cytokines leads to T1D development. That prompted us to study the meaning of plasma constituents of the immune response in BB/OK rats developing T1D and 2 congenic derivatives without T1D (BB.1K; BB.4S) or with diminished T1D frequency (BB.6S). All animals were studied at an age of 4 weeks, some days before initial insulitis is discovered in BB/OK rats. Plasma concentrations of 24 biological markers were assayed using the Bio-Plex Pro ${ }^{\mathrm{TM}}$ Rat cytokine 24plex assay system (order no.171-K1001M; Bio-Rad, Hercules, CA, USA).

Significant differences between $\mathrm{BB} / \mathrm{OK}$ and all other strains were only observed for $\mathrm{M}-\mathrm{Csf}$. BB/OK rats had significantly higher values than BB.6S, BB. $4 \mathrm{~S}$ and $\mathrm{BB} .1 \mathrm{~K}$ rats. In addition, BB.6S rats had significantly reduced M-Csf values compared to BB.1K rats. Obviously significant differences were found in IL-18 and Epo, where samples from BB.6S had the highest values vs all other strains.

M-Csf controls the production, differentiation and function of macrophages. They are the first cells to invade the pancreatic Langerhans islets before T1D onset. The correlation of M-Csf concentration in plasma with T1D development in BB/OK rats and their congenic derivatives may provide evidence that macrophage production is activated in dependence on T1D development at an early age.
\end{abstract}

Keywords: type 1 diabetes, diabetes-prone bb rats, diabetes-resistant congenic bb rats, cytokines, m-CSF, il-18, erythropoietin
Volume I Issue 3 - 2014

\author{
Jorn Lange,' Carina Muller, ${ }^{2}$ Felix Niessner, ${ }^{2}$ \\ Axel Ekkernkamp,' Ingrid Kloting ${ }^{2}$ \\ 'Department of Trauma and Reconstructive Surgery, University \\ of Medicine Greifswald, Germany \\ ${ }^{2}$ Department of Laboratory Animal Science, University of \\ Medicine Greifswald, Germany
}

Correspondence: Ingrid Kloting, Department of Laboratory Animal Science, Institute of Pathophysiology, University of Medicine Greifswald, Greifswalder Str. II, D- 17495 Karlburg, Germany, Tel +49 383 486I 926I, Fax 0383 486I 9 III, Email kloeting@uni-greifswald.de

Received: June 19,2014 | Published: June 26, 2014
Abbreviations: $\mathrm{BB}$, bio breeding; $\mathrm{BB} / \mathrm{OK}$, bio breeding/ ottawa karlsburg; BB.6S, BB.4S and BB.1K, congenic derivates of $\mathrm{bb} / \mathrm{ok}$ with transferred chromosomal region from SHR or KWR; SHR, spontaneously hypertensive rats (SBP 180-240mm hg); KWR, inbred wild rats captured in northern germany; T1D, type 1 diabetes; MHC, major histocompatibility complex; IDDM1/Iddm1, insulin-dependent diabetes mellitus QTL1; Iddm2-Lyp, insulin-dependent diabetes mellitus QTL2-lymphopenia; Gimap5, gtpase imap family member 5; IL-1RA, il-1 receptor antagonist; IL-1a, $-1 \beta,-2,-4,-5,-6,-10,-12 \mathrm{p} 70$, $-13,-7,-17 \mathrm{a}$, and -18 , interleukins $1 \alpha \ldots 18$; Gm-Csf, granulocytemacrophage colony-stimulating factor; G-Csf, granulocyte colonystimulating factor; M-Csf, macrophage colony-stimulating factor; Cxcl1, chemokine (C-X-C) ligand 1; Ifng, interferon gamma; Tnfa, tumor necrosis factor alpha; Epo, erythropoietin; $\mathrm{Ccl} 2, \mathrm{Ccl} 3, \mathrm{Ccl} 5$ and Ccl20, chemokine (C-C motif) ligands 2, 3, 5 and 20; Vegf, vascular endothelial growth factor; Th1 and Th2. T helper 1 or 2

\section{Introduction}

Type 1 diabetes (T1D) is a chronic autoimmune disease in which a loss of tolerance to insulin-producing B-cells in the pancreatic islets results in impaired glucose homeostasis. T1D is characterized by a destruction of pancreatic $\beta$-cells by islet-reactive $T$ cells. Proinflammatory cytokines such as IL- $1 \beta$, IFN- $\gamma$ and TNF- $\alpha$ promote the destruction of pancreatic $\beta$-cells, while regulatory cytokines (IL10 and TGF- $\beta 1$ ) and anti-inflammatory cytokines (IL-1 receptor antagonist [IL-1RA]) avoid $\beta$-cell destruction. An imbalance between pro- and anti-inflammatory and/or regulatory cytokines is essential for the development of T1D. ${ }^{1-3}$

Bio Breeding (BB) rats are widely used as a model for T1D because they display many aspects of disease pathogenesis similar to that found in humans and harbor a general predisposition to autoimmunity, which is modulated by genetic background. In BB rats, the development of diabetes proceeds from an initial phase of insulitis, characterized by $\mathrm{T}$ and $\mathrm{B}$ cell infiltrates in the absence of B-cell damage, to an aggressive stage in which B-cells are destroyed and glucose homeostasis is disrupted.

T1D clusters in families and is frequently associated with other autoimmune disorders, suggesting that an underlying genetic susceptibility compromises tolerance to multiple normal tissues. Extensive linkage analysis of families with T1D and BB rats yielded more than 20 genetic susceptibility loci. Among these, the Major Histocompatibility Complex (MHC) class II locus exerts the most important influence on disease development termed IDDMI in human and IddmI in rats. Several non-MHC related genes have also been implicated. One of them is the gene for lymphopenia in BB rats, termed Iddm 2 (also known as Lyp; Gimap5 and Ian4), which - like Iddm I- is also essential for T1D in BB rats. Nonetheless, multiple additional loci remain to be identified, although characterization of these gene products has been hampered by the large number of immune defects associated with the disease and a limited understanding of the key pathogenic mechanisms. $^{4}$ 
One of the diabetes-prone $\mathrm{BB}$ rat strains is the $\mathrm{BB} / \mathrm{OK}$ rat, which belongs to the best-characterized $\mathrm{BB}$ rat subline in the world. ${ }^{5}$ In addition, several congenic $\mathrm{BB} / \mathrm{OK}$ rat strains with varying diabetes frequency have been developed..$^{5-8}$ To elucidate the meaning of plasma constituents of immune response, we studied 24 plasma constituents of $\mathrm{BB} / \mathrm{OK}$ rats and 2 congenic derivatives without T1D development, lacking one essential diabetogenic gene $I d d m 1(\mathrm{BB} .1 \mathrm{~K} ;)^{7}$ or $I d d m 2$ (BB.4S;). ${ }^{6}$ Furthermore, one congenic BB/OK strain developing T1D at a frequency of $15 \%$ (BB.6S) was used. This congenic BB/ OK strain develops such a low diabetes frequency despite the fact that it possesses both essential diabetogenic genes Iddm 1 and $\operatorname{Iddm} 2{ }^{8}$ All animals were studied at an age of 4 weeks, some days before first insulitis is discovered in $\mathrm{BB} / \mathrm{OK}$ rats.

\section{Materials and methods}

\section{Animals}

All rats were bred and kept in our own animal facility under strict hygienic conditions. All rats had free access to food and acidulated water. All experiments were performed in accordance with the regulations for animal care of the Ministry of Nutrition, Agriculture and Forestry of the Government of Mecklenburg-Vorpommern (Germany). BB/OK was used as an animal model for T1D and its congenic derivatives generated with varying diabetes frequency, as shown in (Table 1). ${ }^{10}$

\section{Determination of cytokines}

Plasma concentrations of 24 biological markers were assayed in rats using the Bio-Plex Pro ${ }^{\mathrm{TM}}$ Rat cytokine 24-plex assay system (order no.171-K1001M; Bio-Rad, Hercules, CA, USA), which allowed simultaneous identification of cytokines in a 96-well filter plate, as described recently: ${ }^{11}$ interleukins(IL-1a, $-1 \mathrm{~b},-2,-4,-5,-6$, $-10,-12 p 70,-13,-7,-17 \mathrm{a}$ and -18$)$, granulocyte-macrophage colonystimulating factor(Gm-Csf), granulocyte colony-stimulating factor(GCsf), macrophage colony-stimulating factor(M-Csf), chemokine(C$\mathrm{X}-\mathrm{C})$ ligand 1(Cxcl1), interferon gamma(Ifng), tumor necrosis factor alpha(Tnfa), Erythropoietin(Epo), chemokine(C-C motif) ligand 2(Ccl2), 3(Ccl3), 5(Cc15) and 20(Ccl20) and vascular endothelial growth factor (Vegfa). The range of detection for all target cytokines was $2-32,000 \mathrm{pg} / \mathrm{mL}$.

Table I Whole litters of BB/OK $(n=10), B B \cdot 6 S(n=I I), B B .4 S(n=9)$ and BB.IK(n=6) born on the same day were used at an age of 4 weeks. Blood samples were obtained from non-fasting rats by puncturing the ophthalmic venous plexus to determine plasma constituents of the immune system

\begin{tabular}{llllll}
\hline Strain & $\begin{array}{l}\text { Transferred } \\
\text { Chromosomal Regions }\end{array}$ & Donor Strain & Diabetes-Frequency (\%) & $\begin{array}{l}\text { Rat Genome } \\
(\mathbf{2 6 0 0 M b}))^{10}\end{array}$ & Rat Genome (\%) \\
\hline BB/OK & Parental strain & & 100 & 0 & 0 \\
BB.6S & $6 q 32$ & SHR & 10 & 33 & 1,3 \\
BB.4S & $4 q 22 /$ Lyp/lddm2 & SHR & 0 & 57,3 & 2,2 \\
BB.IK & 20p/MHC/lddmI & SHR & 0 & 3 & 0,1 \\
\hline
\end{tabular}

\section{Statistical analysis}

Data are given as mean \pm SD. Differences were assessed by oneway analysis of variance with the Bonferroni-Holm correcting using the statistical analysis software SPSS (SPSS Inc., Chicago, IL, USA).

\section{Results}

As shown (Figure 1), there were no significant differences between strains in terms of Il-1a, 4, 10, 17a, G-Csf, and Ccl20. Il-12p70 was only detectable in 3 samples of $\mathrm{BB} / \mathrm{OK}$ rats and in no sample of the other strains, whereas $\mathrm{Ccl} 3$ was detectable in 2 out of 10 samples in BB/OK, in all samples of BB.6S (11/11) and in 3 out of 9 samples of BB.4S. In BB.1K samples, no $\mathrm{Ccl} 3$ was detectable. Significant differences between $\mathrm{BB} / \mathrm{OK}$ and all other strains were only observed for $\mathrm{M}-\mathrm{Csf}$. BB/OK rats had significantly higher values than BB.6S, BB. 4 S and BB. $1 \mathrm{~K}$ rats. In addition, BB.6S rats had significantly reduced M-Csf values compared to BB.1K rats. Furthermore, BB/ OK rats also showed significantly higher values of Il-1b, Il-5, Il-6, Il-13, and Gm-Csf than did BB.4S rats (BB/OK>BB.4S). For Il-7 and $\mathrm{Cxcl1}, \mathrm{BB} / \mathrm{OK}$ rats had higher values than did BB.6S and BB.1K (BB/ $\mathrm{OK}>\mathrm{BB} .6 \mathrm{~S}$ and BB.1K), whereas for Infg, Tnfa, and Vegfa, BB/OK rats showed higher values than did BB.6S and BB.4S $(\mathrm{BB} / \mathrm{OK}>\mathrm{BB} .6 \mathrm{~S}$ and BB.4S). In contrast to all other plasma constituents, $\mathrm{Ccl} 5$ is significantly increased in $\mathrm{BB} / \mathrm{OK}$ and $\mathrm{BB} .4 \mathrm{~S}$ compared to $\mathrm{BB} .6 \mathrm{~S}$ and BB.1K. Only in $\mathrm{Ccl} 2$ were significant differences between BB/OK and BB.6S observed. Obviously significant differences were found in IL-18 and Epo, where samples of BB.6S had the highest values of all strains. Therefore, Epo and Il-18 are highly correlated ( $\mathrm{r}=0.957$; $\mathrm{p}<0.0001$ ). In addition, there were only two significant correlations between T1D frequency and plasma concentrations for M-Csf $(0.743$, $\mathrm{p}<0.0001)$ and $\operatorname{Vegfa}(0.698, \mathrm{p}<0.0001)$.
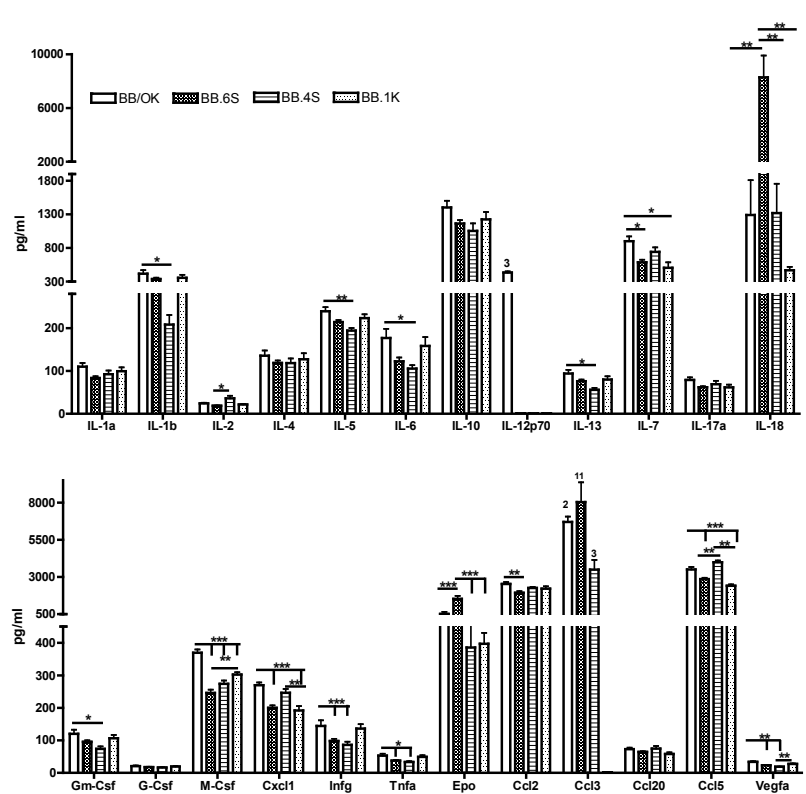

Figure I Plasma constituents of $\mathrm{BB} / \mathrm{OK}$ rats and their congenic derivatives. $\left(p<0.05^{*} ; p<0.0 I^{* *} ; p<0.00 I^{* * *}\right)$

\section{Discussion}

In T1D, the insulin producing ß-cells are destroyed by an imbalance between pro- and anti-inflammatory and/or regulatory cytokines. This prompted us to study the cytokine profile in the plasma of diabetesprone $\mathrm{BB} / \mathrm{OK}$ rats and three congenic strains partially (BB.6S) or totally protected (BB.4S; BB.1K) from T1D development. 
As demonstrated (Figure 1), the anti-inflammatory cytokines such as Il-2, Il-4 and Il-10 indicate no preventive association with T1D, because their concentrations showed no significant differences between $\mathrm{BB} / \mathrm{OK}$ and their congenic derivatives. Moreover, Infg and Tnfa as pro-inflammatory cytokines are significantly increased in BB/ OK, developing to $100 \%$ T1D frequency in comparison to BB.6S and BB.4S, whose T1D frequency was $10 \%$ and $0 \%$, but there is no significant difference between BB. $1 \mathrm{~K}$ and BB.4S, both of which did not develop T1D. Therefore it seems that the classical cytokines thought to be essential for the development of T1D do not play an important role in diabetes in these rat strains at an age of 4weeks. ${ }^{1}$ In contrast, obvious correlations were observed between T1D and M-Csf as well as Vegfa, whereas IL-18, Epo and $\mathrm{Ccl} 3$ were found to be very significant factors for the reduction of T1D in congenic BB.6S.

M-Csf controls the production, differentiation and function of macrophages. They are the first cells to invade pancreatic Langerhans islets before T1D onset. The correlation of M-Csf concentration in plasma with T1D development in $\mathrm{BB} / \mathrm{OK}$ rats and their congenic derivatives may provide evidence that macrophage production is activated in dependence on T1D development at an early age. Because Vegfa is stimulated by M-Csf, the correlation between T1D and Vegfa is, therefore, not unexpected. ${ }^{12}$

The highest values of Il-18 and Epo were found in BB.6S. Il-18 is the only cytokine with a unique capacity to induce T helper 1 (Th1) or Th2 polarization, depending on the immunologic context, ${ }^{13}$ and Epo exerts cytoprotective effects. ${ }^{14}$ In our study, it seems that both Il-18 and Epo have a diabetes-protective effect in BB.6S rats, because only $10 \%$ of them develop T1D despite being homozygous for the essential diabetogenic genes Iddm1 and Iddm2. $\mathrm{Ccl} 3$ showed the highest diabetes-protective effect, which occurred in all BB.6S rats at a similarly high level, whereas $\mathrm{Ccl} 3$ in the other strains was detectable in the plasma of only a few (BB/OK, BB.4S) or no rats (BB.1K). This can be supported by the fact that $\mathrm{Ccl} 3$ in knockout mice which do not produce $\mathrm{Ccl} 3$ showed reduced destructive insulitis and were protected from diabetes. ${ }^{15}$

\section{Conclusion}

The results of this study indicate that BB.6S rats must have completely different mechanisms leading to diabetes protection than are present in $\mathrm{BB} .4 \mathrm{~S}$ or $\mathrm{BB} .1 \mathrm{~K}$. If this finding is true, $\mathrm{BB} / \mathrm{OK}$ should be protected by the $\mathrm{Ccl} 3$ deficiency observed here, but they develop up to $100 \%$ T1D up to an age of 30 weeks. Furthermore, histological studies of BB.6S rats have shown that more than $50 \%$ of islets were infiltrated at an age of about 30 days, whereas $\mathrm{BB} / \mathrm{OK}$ rats showed an infiltration rate of only about $5 \% .^{9}$ This may mean that the islet infiltration in BB.6S is a benign form which should now be supported by high IL-18 and Epo concentration in plasma at 4weeks of age.

\section{Acknowledgements}

We thank Silvia Sadewasser for expert technical assistance. This research is partially supported by Else Kroner-Fresenius-Stiftung 2011 A62.

\section{Conflict of interest}

The authors declare that there is no conflict of interest.

\section{References}

1. Rabinovitch A, Suarez-Pinzon WL. Cytokines and their roles in pancreatic islet beta-cell destruction and insulin dependent diabetes mellitus. Biochem Pharmacol. 1998;55(8):1139-1149.

2. Donath MY, Storling J, Berchtold LA, et al. Cytokines and betacell biology: from concept to clinical translation. Endocr Rev. 2008;29(3):334-350.

3. Bettini M, Vignali DA. T cell-driven initiation and propagation of autoimmune diabetes. Curr Opin Immunol. 2011;23(6):754-760.

4. Acharjee S, Ghosh B, Al-Dhubiab BE, et al. Understanding type 1 diabetes: etiology and models. Can J Diabetes. 2013;37(4):269-276.

5. http://www.medizin.uni-greifswald.de/labanim/available_rat.html

6. Kloting I, Kovacs P, Kuttler B. Phenotypic consequences after restoration of lymphopenia in the diabetes-prone BB/OK rat. Biochem Biophys Res Commun. 1997;239(1):106-110.

7. Kloting I, Voigt B, Kovacs P. Metabolic features of newly established congenic diabetes-prone BB.SHR rat strains. Life Sci. 1998;62(11):973-979.

8. Kloting I, van den Brandt J, Kuttler B. Genes of SHR rats protect spontaneously diabetic $\mathrm{BB} / \mathrm{OK}$ rats from diabetes: Lessons from congenic BB.SHR rat strains. Biochem Biophys Res Commun. 2001;283(2):399-405.

9. Lucke S, Kloting I, Pusch A, et al. Endocrine pancreas histology of congenic $\mathrm{BB}$ rat strains with reduced diabetes incidence after genetic manipulation on chromosome 4, 6 and X. Autoimmunity. 2003;36(3):143-149.

10. Gibbs RA, Weinstock GM, Metzker ML, et al. Genome sequence of the Brown Norway rat yields insights into mammalian evolution. Nature. 2004;428(6982):493-521.

11. Houser B. Bio-Rad's Bio-Plex ${ }^{\circledR}$ suspension array system, xMAP technology overview. Arch Physiol Biochem. 2012;118(4):192-196.

12. Eubank TD, Galloway M, Montague CM, et al. M-CSF Induces Vascular Endothelial Growth Factor Production and Angiogenic Activity From Human Monocytes. J Immunol. 2003;171(5):2637-2643.

13. Nakanishi $\mathrm{K}$, Yoshimoto $\mathrm{T}$, Tsutsui $\mathrm{H}$, et al. Interleukin-18 is a unique cytokine that stimulates both Th1 and Th2 responses depending on its cytokine milieu. Cytokine Growth Factor Rev. 2001;12(1):5-72.

14. Choi D, Schroer SA, Lu SY, et al. Erythropoietin protects against diabetes through direct effects on pancreatic beta cells. J Exp Med. 2010;207(13):2831-2842.

15. Cameron MJ, Arreaza GA, Grattan M, et al. Differential Expression of CC Chemokines and the CCR5Receptor in the Pancreas Is Associated with Progression to Type I Diabetes. J Immunol. 2000;165(2):1102-1110. 